\title{
Neutrino oscillation research with KM3NeT/ORCA
}

\section{The KM3NeT Collaboration}

\$ https://www.km3net.org/km3net-author-list-for-icrc-2019

E-mail: b.strandberg@nikhef.nl

The KM3NeT collaboration is currently building the next-generation large-volume $\left(\mathrm{km}^{3}\right.$-size) water-Cherenkov neutrino telescopes at the bottom of the Mediterranean sea. The denser of the two detectors under construction is called KM3NeT/ORCA, which is optimised for the detection of atmospheric neutrinos with energies above $\sim 1 \mathrm{GeV}$. The main research target of the $\mathrm{KM} 3 \mathrm{NeT} / \mathrm{ORCA}$ detector is the measurement of the neutrino mass ordering and atmospheric neutrino oscillation parameters. The projected sensitivity of the detector to the neutrino mass ordering is shown, alongside prospects for early analyses of data collected with a small sub-array of the detector during construction phase.

Corresponding authors: Steffen Hallmann ${ }^{2}$, Bruno Strandberg ${ }^{\dagger 1}$

${ }^{1}$ Nikhef - Nationaal instituut voor subatomaire fysica, Amsterdam, Netherlands

${ }^{2}$ Friedrich-Alexander-Universität Erlangen-Nürnberg, ECAP, Erlangen, Germany

36th International Cosmic Ray Conference -ICRC2019-

July 24th - August 1st, 2019

Madison, WI, U.S.A.

\footnotetext{
* for collaboration list see PoS(ICRC2019)1177

${ }^{\dagger}$ Speaker.
} 


\section{Overview}

The KM3NeT collaboration is building the next-generation large-volume neutrino telescopes at the bottom of the Mediterranean sea at $\sim 3 \mathrm{~km}$ depth. The detectors are constructed as 3dimensional grids of Digital Optical Modules (DOMs), each DOM housing 31 photomultiplier tubes (PMTs) for the detection of Cherenkov light emitted by charged particles emerging from from neutrino interactions in sea water. The DOMs are attached to Detection Units (DUs), which are vertical strings anchored to the seabed with cabling for power supply and data read-out from the DOMs to the shore-station. Each DU holds 18 DOMs, and a detector consists of 115 DUs. The spacing between the DOMs on a DU and the DUs themselves is configurable and the construction of two detectors with different DOM-density is currently being pursued. The KM3NeT/ARCA detector is being built off-coast Italy and will instrument $\sim 1 \mathrm{~km}^{3}$ of sea water, with the main physics goal of detection of TeV-scale neutrinos from cosmic sources. The KM3NeT/ORCA detector is being built off-coast France and will instrument $\sim 5.5 \times 10^{-3} \mathrm{~km}^{3}$ of sea water with smaller spacing between the DOMs compared to KM3NeT/ARCA, with the main physics goal of determining the neutrino mass ordering (NMO). More details concerning the two detectors can be found in $[1,2]$. This contribution focuses on the KM3NeT/ORCA detector, its sensitivity to the NMO and other oscillation phenomena.

Access to oscillation physics is made possible by the detection of atmospheric neutrinos that traverse Earth and interact within or in close vicinity of the KM3NeT/ORCA instrumented volume. The energy, direction and flavour (separation between track-like and cascade-like events) of a neutrino are reconstructed from the light patterns registered with the detector and accumulated in two-dimensional histograms with neutrino energy on one axis and neutrino zenith direction on the other axis. Different oscillation phenomena lead to different expectation-value distributions in such histograms. Comparison of the experimental data with model predictions facilitates the determination of the NMO and oscillation parameter values, and also allows to test various Beyond the Standard Model (BSM) theories. A detailed overview of the analysis for NMO measurement with $\mathrm{KM} 3 \mathrm{NeT} / \mathrm{ORCA}$ is available in [1].

The first KM3NeT/ORCA lines were deployed in 2017. Currently four DUs have been successfully deployed and are collecting data. The first analysis of the data has been performed for the measurement of atmospheric muon rate dependence [2], as well as for the detection of the first neutrino candidates with KM3NeT [3].

\section{KM3NeT/ORCA sensitivity to the NMO}

A new study for the sensitivity of the KM3NeT/ORCA detector towards the measurement of the NMO has been performed. The projections are based on a new Monte-Carlo production that uses a detector geometry with $20 \mathrm{~m}$ average horizontal spacing and $9 \mathrm{~m}$ vertical spacing between the DOMs, compared to the $23 \mathrm{~m} \times 9 \mathrm{~m}$ geometry used for the results in Ref. [4]. This configuration has been shown to be slightly more favorable for the NMO measurement with KM3NeT/ORCA [1] and is considered technically feasible after the expertise gained from detector deployment.

For this study, average datasets (often referred to as "Asimov" datasets) corresponding to 3 years of data taking were created. Each dataset represents the average number of neutrino events 
expected in each bin of a two-dimensional histogram with reconstructed neutrino energy on one axis and reconstructed neutrino zenith direction on the other axis, as would be obtained by taking the average of $N \gg 1$ experiments. The average datasets were created for both NMO hypotheses (normal-ordered (NO) and inverted-ordered (IO)) with $\delta_{C P}=0$ and $\delta_{C P}=\pi$ at different $\theta_{23}$ values in the range $\theta_{23} \in\left[40^{\circ}, 50^{\circ}\right]$. Other oscillation parameters were set to the NuFit 3.2 values from Ref. [5]. Each dataset was fitted assuming the opposite ordering (e.g. normal-ordered dataset was fitted with an inverted-ordered model), which enabled the calculation of the log-likelihood ratio to establish the sensitivity towards the NMO. The oscillation parameters $\Delta m_{21}^{2}$ and $\theta_{12}$ were fixed during fitting and $\theta_{13}$ was assigned a prior from Ref. [5], as they are very strongly constrained from world data compared to the sensitivity that could be obtained with KM3NeT/ORCA for these parameters. The other three oscillation parameters $\delta_{C P}, \Delta m_{31}^{2}$ and $\theta_{23}$ were left free and unconstrained. Each dataset was fitted twice, with $\theta_{23}$ starting value in different octants, to resolve the global maximum in the likelihood landscape with respect to $\theta_{23}$. Additionally, a set of flux, crosssection and normalisation systematics were used. The configuration of all of the parameters is summarised in Table 1, center column.

For fitting, each dataset was divided into subsets, based on the particle identification (PID) score for flavour separation. The PID is based on a Random Decision Forest (RDF) classifier that is trained on Monte-Carlo data to separate track-like events from shower-like events on a scale from 0 (shower-like) to 1 (track-like). Recently, new features were added for the training of the classifier, comparing hit/no-hit information in the PMTs around the reconstructed position and direction of

\begin{tabular}{|l|l|l|}
\hline \hline Parameter & fixed/prior/free ORCA & fixed/prior/free sub-array \\
\hline \hline$\Delta m_{21}^{2}$ & fixed to $7.4 \cdot 10^{-5} \mathrm{eV}^{2}$ & fixed to $7.4 \cdot 10^{-5} \mathrm{eV}^{2}$ \\
\hline $\sin ^{2} \theta_{12}$ & fixed to $33.62^{\circ}$ & fixed to $33.62^{\circ}$ \\
\hline $\sin ^{2} \theta_{13}$ & $8.54^{\circ}, 0.15^{\circ}$ prior (NO), & fixed to $8.54^{\circ}$ \\
\hline$\delta_{C P}$ & $8.58^{\circ}, 0.14^{\circ}$ prior (IO) & \\
\hline flux $v_{\mu} \leftrightarrow \bar{v}_{\mu}$ skew & free & fixed to $234^{\circ}$ \\
\hline flux $v_{e} \leftrightarrow \bar{v}_{e}$ skew & $10 \%$ prior & $10 \%$ prior \\
\hline flux $v_{\mu} \leftrightarrow v_{e}$ skew & $10 \%$ prior & $10 \%$ prior \\
\hline flux $E$-tilt & $10 \%$ prior & $10 \%$ prior \\
\hline flux $\cos \theta$-tilt & free & free \\
\hline NC cross-sec. norm. & free & free \\
\hline norm. track-like & $10 \%$ prior & $10 \%$ prior \\
\hline norm. shower-like & free & free \\
\hline norm. middle sample & free & free \\
\hline \hline
\end{tabular}

Table 1: Configuration of the oscillation and systematic parameters for the NMO analysis with the full ORCA detector of Sect. 2 (center column) and for the early oscillation analysis with a small ORCA sub-array of Sect. 3.1 (right column). Gaussian priors with the indicated $1 \sigma$ limits were used. 


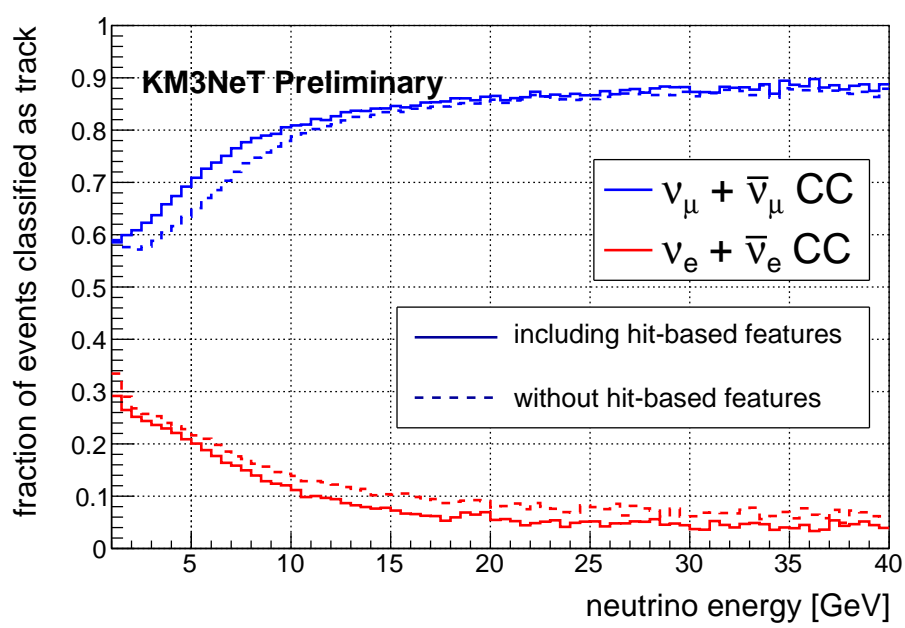

Figure 1: PID performance improvement due to new features for event classification.

the particle with the expected distribution from charged-current (CC) $v_{\mu}$ and $v_{e}$ events. This led to an improvement in the performance of the classifier, as illustrated in Fig. 1. The figure depicts the fraction of $v_{\mu}$ and $v_{e}$ events classified as track-like (PID score $>0.5$ in this case) with and without the new features. The performance of the classifier improves for low-energy $v_{\mu}$ events and for $v_{e}$ events over a wide range of energies. The enhancement in the low-energy region is the most significant, as the energy range $\sim 5-15 \mathrm{GeV}$ is important for the NMO measurement. Further improvements in the future could be seen through the use of a deep-learning event classifier and reconstruction algorithms for KM3NeT/ORCA [6].

The PID score was employed to divide each dataset into strongly shower-like events, strongly track-like events, and a middle-sample, whereas in the previous analysis [4] only two PID classes were used. Additionally, compared to Ref. [4] an improved energy-estimate was used for the

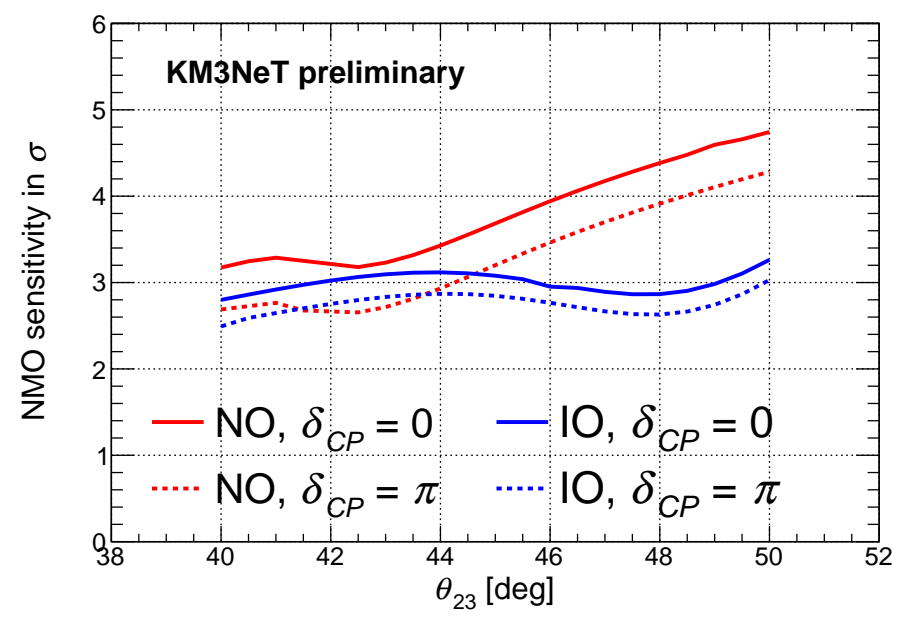

Figure 2: NMO sensitivity of the full KM3NeT/ORCA detector after 3 years. 
track-like sample of events, which enhanced the energy resolution for low-energy muon events. The three subsets were fitted simultaneously under the assumption of the opposite ordering for the determination of the NMO sensitivity. The results are depicted in Fig. 2. As has been shown before $[1,4]$, the highest sensitivity for the rejection of the opposite ordering is seen if normal ordering with $\delta_{C P}=0$ and $\theta_{23}$ in second octant is realised in nature, whereas the lowest sensitivity is observed for inverted ordering with $\delta_{C P}=\pi$ and $\theta_{23}$ in first octant.

Several other Monte-Carlo studies are being carried out with the KM3NeT/ORCA detector that are related to oscillation measurements. BSM physics is explored in the analyses for the sensitivity to non-standard interactions [7] and sterile neutrinos [8]. Very recently a letter of interest has been submitted [9] for a neutrino-beam experiment from Protvino accelerator laboratory near Moscow to the KM3NeT/ORCA detector location for the measurement of the $\delta_{C P}$ phase.

\section{Early physics projections}

In addition to the NMO analysis with the full KM3NeT/ORCA detector discussed in Sect. 2, Monte-Carlo studies have been performed for early physics projections with a small sub-array of 7 KM3NeT/ORCA DUs after 1 year of data taking (referred to as ORCA7).

\subsection{Sensitivity to $\Delta m_{32}^{2}$ and $\theta_{23}$}

For this analysis an average dataset corresponding to 1 year of data taking with the 7-DU KM3NeT/ORCA detector was created. The dataset was created at oscillation parameter values from Ref. [5]. Similarly to the analysis of Sect. 2, the dataset was divided into 3 subsets based on the PID score. The three datasets were fitted simultaneously to extract the $90 \%$ confidence level contour in the $\left(\Delta m_{32}^{2}, \theta_{23}\right)$ plane for ORCA7, depicted in Fig. 3 alongside world data and the full KM3NeT/ORCA projections after 3 years of data taking. The oscillation and systematic parameter

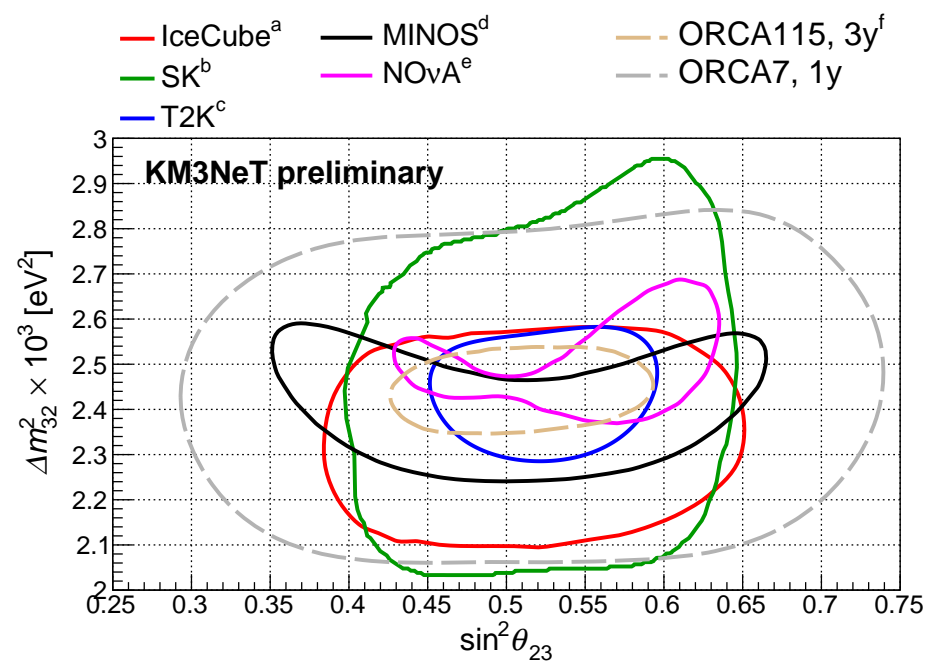

Figure 3: The $90 \%$ confidence level contour in $\left(\Delta m_{32}^{2}, \sin ^{2} \theta_{23}\right)$ plane of the full ORCA detector after 3 years (ORCA115) and ORCA sub-array after 1 year (ORCA7) of data taking, plotted alongside world data. Reference map: a [10], b [11], c [12], d [13], e [14], f [4]. 
configuration for this analysis is summarised in Table 1, right column. Oscillation parameters other than $\Delta m_{32}^{2}$ and $\theta_{23}$ were fixed, as the Monte-Carlo studies indicated ORCA7 to only be marginally sensitive to them. From the systematic parameters, the overall normalisations for the three subsets had the strongest effect on the constraining power for $\Delta m_{32}^{2}$ and $\theta_{23}$, followed by the flux skew systematics. The dominant sensitivity to the atmospheric oscillation parameters with ORCA7 originates from the subset of track-like events. As can be seen in Fig. 3, the projected contour of ORCA7 in $\left(\Delta m_{32}^{2}, \theta_{23}\right)$ plane after 1 year of data taking is somewhat larger compared to the world data, but does suggest that early KM3NeT/ORCA measurements could start providing additional input to global oscillation analyses, such as carried out by the NuFit collaboration [5].

\subsection{Sensitivity to $v_{\tau}$ appearance}

The sensitivity of ORCA7 towards $v_{\tau}$ CC normalisation has also been investigated, which scales the expected number of $v_{\tau} \mathrm{CC}$ interactions. This parameter is of interest, as deviations from the nominal value $N_{\tau}^{\mathrm{cc}}=1$ could indicate physics outside the $3 \times 3$ oscillation model and provide a model-independent hint for new physics, or represent evidence of significant deviations from the predicted cross-section for $v_{\tau} \mathrm{CC}$ interactions. For this study, average datasets were created corresponding to different data accumulation periods, under the hypothesis that the normalisation for $v_{\tau}$ $\mathrm{CC}$ events is $N_{\tau}^{\mathrm{cc}}=1$. For each dataset a profile-likelihood scan in $N_{\tau}^{\mathrm{cc}}$ was performed, marginalising over a set of oscillation and systematic parameters (details of the parameter configuration can be found in Ref. [15]). This enabled the extraction of the exclusion bands for $N_{\tau}^{\mathrm{cc}}$ values that are depicted in Fig. 4. The figure indicates that ORCA7 can exclude $N_{\tau}^{\mathrm{cc}}=0$ at $3 \sigma$ significance after approximately 6 months of data taking. The ORCA7 1-year projections indicate sensitivity for $N_{\tau}^{\mathrm{cc}}$ comparable to the recent results from Ref. [16] and highlight the prospects of early measurements with KM3NeT/ORCA already during the construction phase. The sensitivity of the full KM3NeT/ORCA detector with 115 lines to $N_{\tau}^{\text {cc }}$ has been shown in Ref. [17].

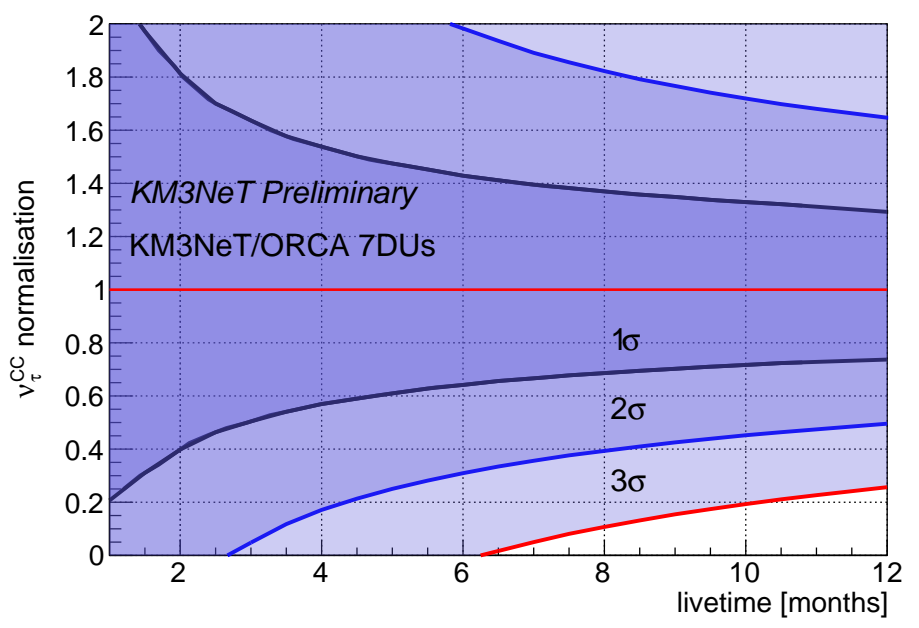

Figure 4: The sensitivity to $N_{\tau}^{\mathrm{cc}}$ of the ORCA sub-array (ORCA7), depending on the data accumulation period. The $1 \sigma, 2 \sigma$ and $3 \sigma$ lines indicate the regions that can be excluded with the corresponding significance under the hypothesis $N_{\tau}^{\mathrm{cc}}=1$ in nature. 


\section{Summary}

A brief overview of the current status of the KM3NeT project has been provided. Updated sensitivity to the NMO with the KM3NeT/ORCA detector after 3 years of data taking has been shown. Early physics projections with a small KM3NeT/ORCA sub-array, consisting of 7 DUs, after 1 year of data taking have been presented, with a focus on $\Delta m_{32}^{2}$ and $\theta_{23}$ measurement and sensitivity to $v_{\tau}$ appearance.

\section{References}

[1] S. Adrian-Martinez and others, J. Phys. G 43, 084001 (2016).

[2] M. Ageron and others, arXiv:1906.02704 (2019).

[3] J. Hofestädt, on behalf of the KM3NeT collaboration, PoS(ICRC2019)910 (2019).

[4] S. Bourrett and L. Quinn, on behalf of the KM3NeT collaboration, DOI: 10.5281/zenodo.1300771 (2018).

[5] I. Esteban and others, JHEP 01, 87 (2017).

[6] M. Moser, on behalf of the KM3NeT collaboration, PoS(ICRC2019)904 (2019).

[7] N. Chowdhury, on behalf of the KM3NeT collaboration, PoS(ICRC2019)931 (2019).

[8] A. Domi, on behalf of the KM3NeT collaboration, PoS(ICRC2019)870 (2019).

[9] A. V. Akindinov and others, arXiv:1902.06083 (2019).

[10] M. G. Aartsen and others, Phys. Rev. Lett. 120, 071801 (2018).

[11] K. Abe and others, Phys. Rev. D 97, 072001 (2018).

[12] M. Wascko, DOI: 10.5281/zenodo.1286752 (2018).

[13] A. Aurisano, DOI: 10.5281/zenodo.1286760 (2018).

[14] M Sanchez, DOI: 10.5281/zenodo.1286758 (2018).

[15] T. Eberl, S. Hallmann and J. Hofestädt, on behalf of the KM3NeT collaboration, PoS(ICRC2017)1025 (2017).

[16] M. G. Aartsen and others, Phys. Rev. D. 99, 032007 (2019).

[17] T. Eberl, S. Hallmann and J. Hofestädt, on behalf of the KM3NeT collaboration, DOI: 10.5281/zenodo.1292823 (2018). 